\title{
Robust Adaptive Control Design for Nonlinear Systems with Uncertainty Based on Backstepping Method
}

\author{
Chiharu Ishii ${ }^{\dagger}$ and Tielong Shen ${ }^{\dagger \dagger}$ \\ $\dagger$ Ashikaga Institute of Technology, Oomaecho 268-1, Ashikagashi, Tochigi, Japan \\ E-mail: c-ishii@ashitech.ac.jp \\ †† Sophia University, Kioicho 7-1, Chiyoda-ku, Tokyo, Japan \\ E-mail: tetu-sin@hoffman.cc.sophia.ac.jp
}

Abstract

This paper discusses a design method of a robust adaptive stabilizing controller for nonlinear systems with uncertainty. The control scheme proposed in [5] for nonlinear systems with relative degree 1 is extended to nonlinear systems with relative degree more than 1 . The robust adaptive stabilizing controller is obtained using the control scheme in [5] recursively.

\section{Introduction}

Recently, adaptive control for nonlinear systems has been developed using the approach called "backstepping" [1]. This technique is lying the notion of stabilizing method for nonlinear systems based on passivity [2]. If nonlinear system is passive and storage function is positive definite, the system can be stabilized by feedback control ${ }^{[3]}$. In this case, the storage function plays a role as Lyapunov function. Assuming asymptotic stability of the zero dynamics of nonlinear system, based on its Lyapunov function, such a stabilizing control scheme that construct a Lyapunov function for whole system recursively so as to ensure convergence of the system output to zero and boundedness of the estimated parameter error has been proposed [4], which is the most systematic methodology for adaptive nonlinear control design. In [5], this methodology was extended to the nonlinear systems with uncertainty such as modeling error, and robust adaptive regulation problem was developed, in which both convergence of the system output to zero and boundedness of the estimated parameter error are guaranteed in the presence of uncertainty. However, control scheme proposed in [5] is restricted only for the case where nonlinear systems have relative degree 1 . The purpose of this paper is to extend the control scheme in [5] for the case where nonlinear systems have relative degree more than 1 . This paper gives some conditions which nonlinear systems should satisfy for solvability of the problem. Then, a robust adaptive stabilizing controller can be obtained using control scheme in [5] recursively, in which Tuning Function is utilized to avoid repetition of parameter estimation.

\section{System Description}

Consider the following SISO nonlinear system given by

$$
\left\{\begin{array}{l}
\dot{x}=f(x)+\Delta f(x)+p(x) \theta+g(x) u \\
y=h(x)
\end{array},\right.
$$

where $x \in R^{n}$ is state vector, $u \in R$ is input and $y \in R$ is output of the system. $f, p, g$ and $h$ are smooth known functions satisfying $f(0)=0, p(0)=0$ and $h(0)=0$. $\theta \in R^{q}$ is unknown constant parameter and $\Delta f(x)$ is unknown nonlinear function representing uncertainty such as modeling error. Let $\Delta f(x)$ be described as

$$
\Delta f(x)=e(x) \delta(x), \quad \Delta f(0)=0
$$

where $e: R^{n} \rightarrow R^{n \times m}$ is known vector function and $\delta: R^{n} \rightarrow R^{m}$ is unknown vector function whose gain is bounded by known function $n(x)$, i.e. $\delta(x)$ is assumed to belong to the set $\Omega$ defined by

$$
\Omega=\{\delta(x) \mid\|\delta(x)\| \leq\|n(x)\|\} .
$$

For system (1), assume the following conditions.

A1 $\left\{g(x) a d_{f} g(x) \cdots a d_{f}^{i-1} g(x)\right\}$ is involutive for any $i$ $(i=1,2, \cdots, r)$, and has rank $i$.

A2 Let $p(x)=\left[p_{1}(x) p_{2}(x) \cdots p_{q}(x)\right], a d_{p_{k_{1}}} \mathcal{G}_{i} \subset \mathcal{G}_{i}, i=$ $0,1, \cdots, r-2$ holds for any $k_{1}=(1,2, \cdots, q)$.

A3 Let $e(x)=\left[e_{1}(x) e_{2}(x) \cdots e_{m}(x)\right], a d_{e_{k_{2}}} \mathcal{G}_{i} \subset \mathcal{G}_{i}, i=$ $0,1, \cdots, r-2$ holds for any $k_{2}=(1,2, \cdots, m)$.

Where

$$
\begin{gathered}
\mathcal{G}_{i}=\operatorname{span}\left\{g(x), a d_{f} g(x), \cdots, a d_{f}^{i} g(x)\right\}, \\
a d_{p_{k_{1}}} \mathcal{G}_{i}=\operatorname{span}\left\{a d_{p_{k_{1}}} g(x), a d_{p_{k_{1}}}\left(a d_{f} g(x)\right), \cdots, a d_{p_{k_{1}}}\left(a d_{f}^{i} g(x)\right)\right\}, \\
a d_{e_{k_{2}}} \mathcal{G}_{i}=\operatorname{span}\left\{a d_{e_{k_{2}}} g(x), a d_{e_{k_{2}}}\left(a d_{f} g(x)\right), \cdots, a d_{e_{k_{2}}}\left(a d_{f}^{i} g(x)\right)\right\} .
\end{gathered}
$$

These Assumptions are for transforming system (1) to system with triangular structure.

Robust Adaptive Regulation Problem: Find an adaptive controller such that $y \rightarrow 0$ and boundedness of the estimated parameter error are guaranteed.

\section{Derivation of The Control Law for $r=2$}

For the explanation of the notion of our control design method, we first consider the simple case where system 
(1) has relative degree $r=2$. Then, using suitable change of coordinate

$$
\left[\begin{array}{c}
z \\
y_{1} \\
y_{2}
\end{array}\right]=T(x)=\left[\begin{array}{c}
\varphi(x) \\
h(x) \\
L_{f} h(x)
\end{array}\right]
$$

and state feedback

$$
u=\left\{L_{g} L_{f} h(x)\right\}^{-1}\left\{v-L_{f}^{2} h(x)\right\},
$$

system (1) can be transformed to the following system with triangular structure.

$$
\left\{\begin{array}{l}
\dot{z}=f_{0}\left(z, y_{1}\right)+e_{0}\left(z, y_{1}\right) \delta_{0}\left(z, y_{1}, y_{2}\right)+p_{0}\left(z, y_{1}\right) \theta \\
\dot{y}_{1}=y_{2}+b_{0}\left(z, y_{1}\right) \delta_{0}\left(z, y_{1}, y_{2}\right)+q_{0}\left(z, y_{1}\right) \theta \\
\dot{y}_{2}=v+b_{1}\left(z, y_{1}, y_{2}\right) \delta_{0}\left(z, y_{1}, y_{2}\right)+q_{1}\left(z, y_{1}, y_{2}\right) \theta \\
y=h_{0}\left(z, y_{1}, y_{2}\right)
\end{array},\right.
$$

where

$$
\begin{gathered}
f_{0}\left(z, y_{1}\right)=\frac{\partial \varphi}{\partial x} f(x), e_{0}\left(z, y_{1}\right)=\frac{\partial \varphi}{\partial x} e(x), \\
p_{0}\left(z, y_{1}\right)=\frac{\partial \varphi}{\partial x} p(x), b_{0}\left(z, y_{1}\right)=\frac{\partial h}{\partial x} e(x), \\
q_{0}\left(z, y_{1}\right)=\frac{\partial h}{\partial x} p(x), b_{1}\left(z, y_{1}, y_{2}\right)=\frac{\partial L_{f} h}{\partial x} e(x), \\
q_{1}\left(z, y_{1}, y_{2}\right)=\frac{\partial L_{f} h}{\partial x} p(x), \delta_{0}\left(z, y_{1}, y_{2}\right)=\delta(x), \\
h_{0}\left(z, y_{1}, y_{2}\right)=h(x), x=T^{-1}\left(z, y_{1}, y_{2}\right),
\end{gathered}
$$

and output $y=y_{1}$.

Let bounding function $n\left(T^{-1}\left(z, y_{1}, y_{2}\right)\right)$ for uncertainty $\delta_{0}\left(z, y_{1}, y_{2}\right)$ satisfy

$$
\frac{\partial n}{\partial y_{2}}=0
$$

Then, the bounding function is expressed as

$$
\tilde{n}_{0}\left(z, y_{1}\right)=n\left(T^{-1}\left(z, y_{1}, y_{2}\right)\right) .
$$

Zero dynamics of the system (6) is given by

$$
\dot{z}=f_{*}(z)+e_{*}(z) \delta_{0}\left(z, y_{1}, y_{2}\right)+p_{*}(z) \theta
$$

where $f_{*}(z)=f_{0}(z, 0), e_{*}(z)=e_{0}(z, 0)$ and $p_{*}(z)=$ $p_{0}(z, 0)$. For the zero dynamics (8), assume that $p_{*}(z)=0$ and assume also that system (6) has robust minimum phase property, i.e. there exist positive definite functions $V_{0}(z)>0\left(V_{0}(0)=0\right)$ and $Q_{0}(z)>0$ $\left(Q_{0}(0)=0\right)$ such that

$$
L_{f_{*}} V_{0}(z)+L_{e_{*}} V_{0}(z) \delta_{0}\left(z, y_{1}, y_{2}\right) \leq-Q_{0}(z)
$$

is satisfied for all $\delta_{0} \in \Omega$.

Then, from Lemma 1 in appendix, there exists positive definite function $\lambda_{0}(z)$ satisfying

$$
L_{f_{*}} V_{0}(z)+\frac{\lambda_{0}(z)}{2}\left\|L_{e_{*}}^{T} V_{0}(z)\right\|^{2}+\frac{1}{2 \lambda_{0}(z)}\left\|\tilde{n}_{*}(z)\right\|^{2} \leq-Q_{0}(z)
$$

where $\tilde{n}_{*}(z)=\tilde{n}_{0}(z, 0)$.
Besides, smooth functions $f_{0}, \frac{\partial V_{0}}{\partial z} e_{0}, \frac{\partial V_{0}}{\partial z} p_{0}$ and $\tilde{n}_{0}$ are decomposed ${ }^{[5]}$ as

$$
\begin{aligned}
f_{0}\left(z, y_{1}\right) & =f_{*}(z)+f_{1}\left(z, y_{1}\right) y_{1}, \\
\frac{\partial V_{0}}{\partial z} e_{0}\left(z, y_{1}\right) & =\frac{\partial V_{0}}{\partial z} e_{*}(z)+y_{1}^{T} e_{1}\left(z, y_{1}\right), \\
\frac{\partial V_{0}}{\partial z} p_{0}\left(z, y_{1}\right) & =\frac{\partial V_{0}}{\partial z} p_{*}(z)+y_{1}^{T} p_{1}\left(z, y_{1}\right), \\
\tilde{n}_{0}\left(z, y_{1}\right) & =\tilde{n}_{*}(z)+\tilde{n}_{1}\left(z, y_{1}\right) y_{1} .
\end{aligned}
$$

Consider $\left(z, y_{1}\right)$-subsystem

$$
\left\{\begin{array}{l}
\dot{z}=f_{0}\left(z, y_{1}\right)+e_{0}\left(z, y_{1}\right) \delta_{0}\left(z, y_{1}, y_{2}\right)+p_{0}\left(z, y_{1}\right) \theta \\
\dot{y}_{1}=y_{2}+b_{0}\left(z, y_{1}\right) \delta_{0}\left(z, y_{1}, y_{2}\right)+q_{0}\left(z, y_{1}\right) \theta
\end{array}\right.
$$

For system (11), by regarding output of the system $y$ as $y_{1}$ and input of the system $v$ as $y_{2}$, system (11) has relative degree 1 . Besides, system (11) has robust minimum phase property. Therefore, using the design method proposed in [5], a robust adaptive controller can be obtained for system (11). In fact, applying Lemma 2 in appendix, the stabilizing control law is given by

$$
y_{2}=-c_{0}\left(z, y_{1}, \hat{\theta}\right)-\frac{1}{2} y_{1}:=\alpha_{1}\left(z, y_{1}, \hat{\theta}\right),
$$

where function $c_{0}$ is defined in Lemma 2 .

By taking $y_{2}=\alpha_{1}\left(z, y_{1}, \hat{\theta}\right)+\eta_{1}$, the closed-loop system of (11) is described as

$\dot{\xi}_{1}=F_{1}\left(\xi_{1}, \hat{\theta}\right)+P_{1}\left(\xi_{1}\right) \theta+E_{1}\left(\xi_{1}\right) \delta_{0}\left(\xi_{1}, y_{2}\right)+G_{1}\left(\xi_{1}\right) \eta_{1}$

where $\xi_{1}=\left[\begin{array}{ll}z^{T} & y_{1}^{T}\end{array}\right]^{T}$,

$$
\begin{gathered}
F_{1}\left(\xi_{1}, \hat{\theta}\right)=\left[\begin{array}{c}
f_{0}\left(z, y_{1}\right) \\
\alpha_{1}\left(z, y_{1}, \hat{\theta}\right)
\end{array}\right], P_{1}\left(\xi_{1}\right)=\left[\begin{array}{l}
p_{0}\left(z, y_{1}\right) \\
q_{0}\left(z, y_{1}\right)
\end{array}\right], \\
E_{1}\left(\xi_{1}\right)=\left[\begin{array}{l}
e_{0}\left(z, y_{1}\right) \\
b_{0}\left(z, y_{1}\right)
\end{array}\right], G_{1}\left(\xi_{1}\right)=\left[\begin{array}{l}
0 \\
1
\end{array}\right] .
\end{gathered}
$$

Define positive definite functions $V_{1}$ and $Q_{1}$ as

$$
\begin{aligned}
V_{1}\left(z, y_{1}, \tilde{\theta}\right) & =V_{0}(z)+\frac{1}{2} y_{1}^{T} y_{1}+\frac{1}{2} \tilde{\theta}^{T} \tilde{\theta}, \\
Q_{1}\left(z, y_{1}\right) & =Q_{0}(z)+\frac{1}{2} y_{1}^{T} y_{1} .
\end{aligned}
$$

Then, noting inequality (10), the following inequality is obtained.

$$
\begin{aligned}
& \frac{\partial V_{1}}{\partial \xi_{1}}\left\{F_{1}\left(\xi_{1}, \hat{\theta}\right)+P_{1}\left(\xi_{1}\right) \theta\right\}+\frac{\lambda_{1}\left(\xi_{1}\right)}{2}\left\|L_{E_{1}}^{T} V_{1}\left(\xi_{1}\right)\right\|^{2} \\
& \quad+\frac{1}{2 \lambda_{1}\left(\xi_{1}\right)}\left\|\tilde{n}_{0}\left(z, y_{1}\right)\right\|^{2} \\
&=\frac{\partial V_{0}}{\partial z} f_{*}(z)+\frac{\lambda_{0}(z)}{2}\left\|L_{e_{*}}^{T} V_{0}(z)\right\|^{2}+\frac{1}{2 \lambda_{0}(z)}\left\|\tilde{n}_{*}(z)\right\|^{2} \\
& \quad-y_{1}^{T} c_{0}\left(z, y_{1}, \hat{\theta}\right) \\
& \quad+\frac{\partial V_{0}}{\partial z} f_{1}\left(z, y_{1}\right) y_{1}+y_{1}^{T} p_{1}\left(z, y_{1}\right) \theta+y_{1}^{T} q_{0}\left(z, y_{1}\right) \theta \\
& \quad+\frac{\lambda_{0}(z)}{2} y_{1}^{T}\left\{e_{1}\left(z, y_{1}\right)+b_{0}\left(z, y_{1}\right)\right\}\left\{e_{1}^{T}\left(z, y_{1}\right)+b_{0}^{T}\left(z, y_{1}\right)\right\} y_{1} \\
& \quad+\lambda_{0}(z) \frac{\partial V_{0}}{\partial z} e_{*}(z)\left\{e_{1}^{T}\left(z, y_{1}\right)+b_{0}^{T}\left(z, y_{1}\right)\right\} y_{1} \\
& \quad+\frac{1}{\lambda_{0}(z)} y_{1}^{T} \tilde{n}_{1}^{T}\left(z, y_{1}\right)\left\{\tilde{n}_{*}(z)+\frac{1}{2} \tilde{n}_{1}\left(z, y_{1}\right) y_{1}\right\} \\
& \leq-Q_{1}\left(z, y_{1}\right)+y_{1}^{T} c_{2}\left(z, y_{1}\right) \tilde{\theta}, \quad \forall z, y_{1},
\end{aligned}
$$


where $\lambda_{1}$ is chosen as $\lambda_{1}\left(\xi_{1}\right)=\lambda_{0}(z)$.

Consider the time derivative of $V_{1}\left(z, y_{1}, \tilde{\theta}\right)$ along the trajectory of (13).

$$
\begin{aligned}
\dot{V}_{1} & =\frac{\partial V_{1}}{\partial \xi_{1}}\left\{F_{1}\left(\xi_{1}, \hat{\theta}\right)+P_{1}\left(\xi_{1}\right) \theta\right\} \\
& +\frac{\partial V_{1}}{\partial \xi_{1}}\left\{E_{1}\left(\xi_{1}\right) \delta_{0}\left(\xi_{1}, y_{2}\right)+G_{1}\left(\xi_{1}\right) \eta_{1}\right\}+\tilde{\theta}^{T} \dot{\tilde{\theta}} .
\end{aligned}
$$

Substituting (14) into (15), the following inequality is obtained.

$$
\begin{aligned}
\dot{V}_{1} \leq & -Q_{1}\left(z, y_{1}\right)-\frac{\lambda_{1}\left(\xi_{1}\right)}{2}\left\|L_{E_{1}}^{T} V_{1}\left(\xi_{1}\right)-\frac{1}{\lambda_{1}\left(\xi_{1}\right)} \delta_{0}\left(\xi_{1}, y_{2}\right)\right\|^{2} \\
& -\frac{1}{2 \lambda_{1}\left(\xi_{1}\right)}\left\{\left\|\tilde{n}_{0}\left(z, y_{1}\right)\right\|^{2}-\left\|\delta_{0}\left(\xi_{1}, y_{2}\right)\right\|^{2}\right\}+y_{1}^{T} \eta_{1} \\
& +\tilde{\theta}^{T}\left[\tau_{1}\left(z, y_{1}\right)-\dot{\hat{\theta}}\right], \quad \forall z, y_{1}
\end{aligned}
$$

where $\tau_{1}\left(z, y_{1}\right)=c_{2}^{T}\left(z, y_{1}\right) y_{1}$.

Therefore, if parameter update law is chosen as $\dot{\hat{\theta}}=$ $\tau_{1}\left(z, y_{1}\right)$, regarding $\eta_{1}$ as input of the system (13), the system becomes strictly robust passive. However, to avoid repetition of the parameter estimation, parameter update law is not determined here, and $\tau_{1}\left(z, y_{1}\right)$ is left as a Tuning Function.

Now, consider the whole system (6). By applying change of coordinate

$$
\left\{\begin{array}{l}
\xi_{1}=\left[\begin{array}{c}
z \\
y_{1}
\end{array}\right] \\
\eta_{1}=y_{2}-\alpha_{1}\left(z, y_{1}, \hat{\theta}\right)
\end{array},\right.
$$

$\eta_{1}$-subsystem is expressed as follows.

$$
\begin{aligned}
\dot{\eta}_{1}= & \dot{y}_{2}-\dot{\alpha}_{1}\left(z, y_{1}, \hat{\theta}\right) \\
= & v+b_{1}\left(\xi_{1}, y_{2}\right) \delta_{0}\left(\xi_{1}, y_{2}\right)+q_{1}\left(\xi_{1}, y_{2}\right) \theta \\
& -\frac{\partial \alpha_{1}}{\partial z}\left\{f_{0}\left(\xi_{1}\right)+e_{0}\left(\xi_{1}\right) \delta_{0}\left(\xi_{1}, y_{2}\right)+p_{0}\left(\xi_{1}\right) \theta\right\} \\
& -\frac{\partial \alpha_{1}}{\partial y_{1}}\left\{y_{2}+b_{0}\left(\xi_{1}\right) \delta_{0}\left(\xi_{1}, y_{2}\right)+q_{0}\left(\xi_{1}\right) \theta\right\}-\frac{\partial \alpha_{1}}{\partial \hat{\theta}} \dot{\hat{\theta}} \\
= & v-v_{2}\left(\xi_{1}, y_{2}, \hat{\theta}\right)+w_{2}\left(\xi_{1}, y_{2}, \hat{\theta}\right) \theta \\
& +z_{2}\left(\xi_{1}, y_{2}, \hat{\theta}\right) \delta_{0}\left(\xi_{1}, y_{2}\right)-\frac{\partial \alpha_{1}}{\partial \hat{\theta}} \dot{\hat{\theta}}
\end{aligned}
$$

where

$$
\begin{aligned}
& v_{2}\left(\xi_{1}, y_{2}, \hat{\theta}\right)=\frac{\partial \alpha_{1}}{\partial z} f_{0}\left(\xi_{1}\right)+\frac{\partial \alpha_{1}}{\partial y_{1}} y_{2}, \\
& w_{2}\left(\xi_{1}, y_{2}, \hat{\theta}\right)=q_{1}\left(\xi_{1}, \dot{y}_{2}\right)-\frac{\partial \alpha_{1}}{\partial z} p_{0}\left(\xi_{1}\right)-\frac{\partial \alpha_{1}}{\partial y_{1}} q_{0}\left(\xi_{1}\right), \\
& z_{2}\left(\xi_{1}, y_{2}, \hat{\theta}\right)=b_{1}\left(\xi_{1}, y_{2}\right)-\frac{\partial \alpha_{1}}{\partial z} e_{0}\left(\xi_{1}\right)-\frac{\partial \alpha_{1}}{\partial y_{1}} b_{0}\left(\xi_{1}\right) .
\end{aligned}
$$

Finally, in $\left(\xi_{1}, \eta_{1}\right)$ coordinate, the system is given as

$$
\left\{\begin{array}{c}
\dot{\xi}_{1}=F_{1}\left(\xi_{1}, \hat{\theta}\right)+P_{1}\left(\xi_{1}\right) \theta+E_{1}\left(\xi_{1}\right) \delta_{0}\left(\xi_{1}, y_{2}\right)+G_{1}\left(\xi_{1}\right) \eta_{1} \\
\dot{\eta}_{1}=v-v_{2}\left(\xi_{1}, y_{2}, \hat{\theta}\right)+w_{2}\left(\xi_{1}, y_{2}, \hat{\theta}\right) \theta \\
+z_{2}\left(\xi_{1}, y_{2}, \hat{\theta}\right) \delta_{0}\left(\xi_{1}, y_{2}\right)-\frac{\partial \alpha_{1}}{\partial \hat{\theta}} \dot{\hat{\theta}}
\end{array}\right.
$$

Note that in system (19), regarding output of the system as $\eta_{1}$ and input of the system as $v$, relative degree of the system is 1. Besides, from inequality (14), under the estimated parameter error $\tilde{\theta}=0$ the system has robust minimum phase property. Therefore, applying the design method proposed in [5] once more, the robust adaptive controller can be obtained for system (19).

By taking $v=\alpha_{2}\left(\xi_{1}, y_{2}, \hat{\theta}\right)$, the closed-loop system of (19) is expressed as follows.

$\dot{\xi}_{2}=F_{2}\left(\xi_{2}, \hat{\theta}\right)+P_{2}\left(\xi_{2}, \hat{\theta}\right) \theta+E_{2}\left(\xi_{2}, \hat{\theta}\right) \delta_{0}\left(\xi_{2}\right)+H_{2}\left(\xi_{2}, \hat{\theta}\right) \dot{\hat{\theta}}$

where $\xi_{2}=\left[\begin{array}{ll}\xi_{1}^{T} & \eta_{1}^{T}\end{array}\right]^{T}$,

$$
\begin{gathered}
F_{2}\left(\xi_{2}, \hat{\theta}\right)=\left[\begin{array}{c}
F_{1}\left(\xi_{1}, \hat{\theta}\right)+G_{1}\left(\xi_{1}\right) \eta_{1} \\
\alpha_{2}\left(\xi_{2}, \hat{\theta}\right)-v_{2}\left(\xi_{2}, \hat{\theta}\right)
\end{array}\right], P_{2}\left(\xi_{2}, \hat{\theta}\right)=\left[\begin{array}{c}
P_{1}\left(\xi_{1}\right) \\
w_{2}\left(\xi_{2}, \hat{\theta}\right)
\end{array}\right], \\
E_{2}\left(\xi_{2}, \hat{\theta}\right)=\left[\begin{array}{c}
E_{1}\left(\xi_{1}\right) \\
z_{2}\left(\xi_{2}, \hat{\theta}\right)
\end{array}\right], H_{2}\left(\xi_{2}, \hat{\theta}\right)=\left[\begin{array}{c}
0 \\
-\frac{\partial \alpha_{1}}{\partial \hat{\theta}}
\end{array}\right] .
\end{gathered}
$$

Define positive definite functions $V_{2}$ and $Q_{2}$ as

$$
\begin{aligned}
V_{2}\left(\xi_{1}, \eta_{1}, \tilde{\theta}\right) & =V_{1}\left(\xi_{1}, \tilde{\theta}\right)+\frac{1}{2} \eta_{1}^{T} \eta_{1}, \\
Q_{2}\left(\xi_{1}, \eta_{1}\right) & =Q_{1}\left(\xi_{1}\right)+\frac{1}{2} \eta_{1}^{T} \eta_{1} .
\end{aligned}
$$

Then, the following inequality is obtained.

$$
\begin{aligned}
\frac{\partial V_{2}}{\partial \xi_{2}}\left\{F_{2}\left(\xi_{2}, \hat{\theta}\right)+P_{2}\left(\xi_{2}, \hat{\theta}\right) \theta\right\}+\frac{\lambda_{2}\left(\xi_{2}\right)}{2}\left\|L_{E_{2}}^{T} V_{2}\left(\xi_{2}\right)\right\|^{2} \\
\quad+\frac{1}{2 \lambda_{2}\left(\xi_{2}\right)}\left\|\tilde{n}_{0}\left(z, y_{1}\right)\right\|^{2} \\
=\frac{\partial V_{1}}{\partial \xi_{1}}\left\{F_{1}\left(\xi_{1}, \hat{\theta}\right)+P_{1}\left(\xi_{1}\right) \theta\right\} \\
\quad+y_{1}^{T} \eta_{1}+\eta_{1}^{T} \alpha_{2}\left(\xi_{2}, \hat{\theta}\right)-\eta_{1}^{T} v_{2}\left(\xi_{2}, \hat{\theta}\right)+\eta_{1}^{T} w_{2}\left(\xi_{2}, \hat{\theta}\right) \theta \\
+\frac{\lambda_{2}\left(\xi_{2}\right)}{2}\left\|L_{E_{2}}^{T} V_{2}\left(\xi_{2}\right)\right\|^{2}+\frac{1}{2 \lambda_{2}\left(\xi_{2}\right)}\left\|\tilde{n}_{0}\left(z, y_{1}\right)\right\|^{2} \\
=\frac{\partial V_{1}}{\partial \xi_{1}}\left\{F_{1}\left(\xi_{1}, \hat{\theta}\right)+P_{1}\left(\xi_{1}\right) \theta\right\}+\frac{\lambda_{2}\left(\xi_{2}\right)}{2}\left\|L_{E_{1}}^{T} V_{1}\left(\xi_{1}\right)\right\|^{2} \\
\quad+\frac{1}{2 \lambda_{2}\left(\xi_{2}\right)}\left\|\tilde{n}_{0}\left(z, y_{1}\right)\right\|^{2} \\
\quad+\eta_{1}^{T}\left[\alpha_{2}\left(\xi_{2}, \hat{\theta}\right)+y_{1}-v_{2}\left(\xi_{2}, \hat{\theta}\right)+w_{2}\left(\xi_{2}, \hat{\theta}\right) \hat{\theta}\right. \\
\left.\quad+\lambda_{2}\left(\xi_{2}\right)\left\{\frac{1}{2} z_{2}\left(\xi_{2}, \hat{\theta}\right) z_{2}^{T}\left(\xi_{2}, \hat{\theta}\right) \eta_{1}+z_{2}\left(\xi_{2}, \hat{\theta}\right) E_{1}^{T}\left(\xi_{1}\right) \frac{\partial^{T} V_{1}}{\partial \xi_{1}}\right\}\right] \\
\quad+\eta_{1}^{T} w_{2}\left(\xi_{2}, \hat{\theta}\right) \tilde{\theta} .
\end{aligned}
$$

Choose $\alpha_{2}$ as

$$
\begin{aligned}
& \alpha_{2}\left(\xi_{2}, \hat{\theta}\right) \\
& =-y_{1}-\frac{1}{2} \eta_{1}+v_{2}\left(\xi_{2}, \hat{\theta}\right)-w_{2}\left(\xi_{2}, \hat{\theta}\right) \hat{\theta} \\
& \quad-\lambda_{2}\left(\xi_{2}\right)\left\{\frac{1}{2} z_{2}\left(\xi_{2}, \hat{\theta}\right) z_{2}^{T}\left(\xi_{2}, \hat{\theta}\right) \eta_{1}+z_{2}\left(\xi_{2}, \hat{\theta}\right) E_{1}^{T}\left(\xi_{1}\right) \frac{\partial^{T} V_{1}}{\partial \xi_{1}}\right\} \\
& \quad+\nu_{2}\left(\xi_{2}, \hat{\theta}\right),
\end{aligned}
$$

and $\lambda_{2}$ as $\lambda_{2}\left(\xi_{2}\right)=\lambda_{1}\left(\xi_{1}\right)$, then substituting (14) into (21), the following inequality is obtained.

$$
\begin{aligned}
\frac{\partial V_{2}}{\partial \xi_{2}}\left\{F_{2}\left(\xi_{2}, \hat{\theta}\right)+P_{2}\left(\xi_{2}, \hat{\theta}\right) \theta\right\}+\frac{\lambda_{2}\left(\xi_{2}\right)}{2}\left\|L_{E_{2}}^{T} V_{2}\left(\xi_{2}\right)\right\|^{2} \\
\quad+\frac{1}{2 \lambda_{2}\left(\xi_{2}\right)}\left\|\tilde{n}_{0}\left(z, y_{1}\right)\right\|^{2} \\
\leq-Q_{2}\left(\xi_{1}, \eta_{1}\right)+\tilde{\theta}^{T} \tau_{2}\left(\xi_{2}, \hat{\theta}\right)+\eta_{1}^{T} \nu_{2}\left(\xi_{2}, \hat{\theta}\right)
\end{aligned}
$$


where

$$
\tau_{2}\left(\xi_{2}, \hat{\theta}\right)=\tau_{1}\left(\xi_{1}\right)+w_{2}^{T}\left(\xi_{2}, \hat{\theta}\right) \eta_{1}
$$

and $\nu_{2}\left(\xi_{2}, \hat{\theta}\right)$ is an auxiliary input to compensate estimated parameter error, which is determined later.

Consider the time derivative of $V_{2}\left(\xi_{1}, \eta_{1}, \tilde{\theta}\right)$ along the trajectory of (20). The same as derivation of (16), finally the following inequality is obtained.

$$
\begin{aligned}
\dot{V}_{2}= & \frac{\partial V_{2}}{\partial \xi_{2}}\left\{F_{2}\left(\xi_{2}, \hat{\theta}\right)+P_{2}\left(\xi_{2}, \hat{\theta}\right) \theta\right\} \\
& +\frac{\partial V_{2}}{\partial \xi_{2}}\left\{E_{2}\left(\xi_{2}, \hat{\theta}\right) \delta_{0}\left(\xi_{2}\right)+H_{2}\left(\xi_{2}, \hat{\theta}\right) \dot{\hat{\theta}}\right\}+\tilde{\theta}^{T} \dot{\tilde{\theta}} \\
\leq & -Q_{2}\left(\xi_{1}, \eta_{1}\right)-\frac{\lambda_{2}\left(\xi_{2}\right)}{2}\left\|L_{E_{2}}^{T} V_{2}\left(\xi_{2}\right)-\frac{1}{\lambda_{2}\left(\xi_{2}\right)} \delta_{0}\left(\xi_{2}\right)\right\|^{2} \\
& -\frac{1}{2 \lambda_{2}\left(\xi_{2}\right)}\left\{\left\|\tilde{n}_{0}\left(z, y_{1}\right)\right\|^{2}-\left\|\delta_{0}\left(\xi_{2}\right)\right\|^{2}\right\} \\
& -\eta_{1}^{T} \frac{\partial \alpha_{1}}{\partial \hat{\theta}} \dot{\hat{\theta}}+\eta_{1}^{T} \nu_{2}\left(\xi_{2}, \hat{\theta}\right)+\tilde{\theta}^{T}\left[\tau_{2}\left(\xi_{2}, \hat{\theta}\right)-\dot{\hat{\theta}}\right] .
\end{aligned}
$$

By choosing $\nu_{2}$ as

$$
\nu_{2}\left(\xi_{2}, \hat{\theta}\right)=\frac{\partial \alpha_{1}}{\partial \hat{\theta}} \tau_{2}\left(\xi_{2}, \hat{\theta}\right),
$$

inequality

$$
\begin{aligned}
\dot{V}_{2} \leq & -Q_{2}\left(\xi_{1}, \eta_{1}\right) \\
& -\eta_{1}^{T} \frac{\partial \alpha_{1}}{\partial \hat{\theta}}\left[\tau_{2}\left(\xi_{2}, \hat{\theta}\right)-\dot{\hat{\theta}}\right]+\tilde{\theta}^{T}\left[\tau_{2}\left(\xi_{2}, \hat{\theta}\right)-\dot{\hat{\theta}}\right]
\end{aligned}
$$

is obtained. Hence, choosing the parameter update law as

finally

$$
\dot{\hat{\theta}}=\tau_{2}\left(\xi_{2}, \hat{\theta}\right),
$$

$$
\dot{V}_{2}<0, \quad \forall \xi_{1} \neq 0, \forall \eta_{1} \neq 0
$$

holds.

Reviewing the above design procedure, the robust adaptive stabilizing controller for whole system is obtained by repeating the robust adaptive control scheme for systems with relative degree 1 twice. In this procedure, parameter update law is not determined in each step. By introducing a Tuning Function, feedback control law is constructed recursively in each step. Finally, parameter update law is determined in final step. In each step, if the Tuning Function is chosen as the parameter update law, the closed-loop system of subsystem in its step becomes strictly robust passive. This scheme can be extended to the systems with arbitrary relative degree.

\section{Derivation of The Control Law for $r \geq 2$}

Now, we generalize our control scheme. The result in the previous section is embeded in the one in this section. Consider the case where system (1) has relative degree $r \geq 2$. In this case, using suitable change of coordinate

$$
\left[\begin{array}{c}
z \\
y_{1} \\
y_{2} \\
\vdots \\
y_{r}
\end{array}\right]=T(x)=\left[\begin{array}{c}
\varphi(x) \\
h(x) \\
L_{f} h(x) \\
\vdots \\
L_{f}^{r-1} h(x)
\end{array}\right]
$$

and state feedback

$$
u=\left\{L_{g} L_{f}^{r-1} h(x)\right\}^{-1}\left\{v-L_{f}^{r} h(x)\right\},
$$

system (1) can be transformed to the following system with triangular structure.

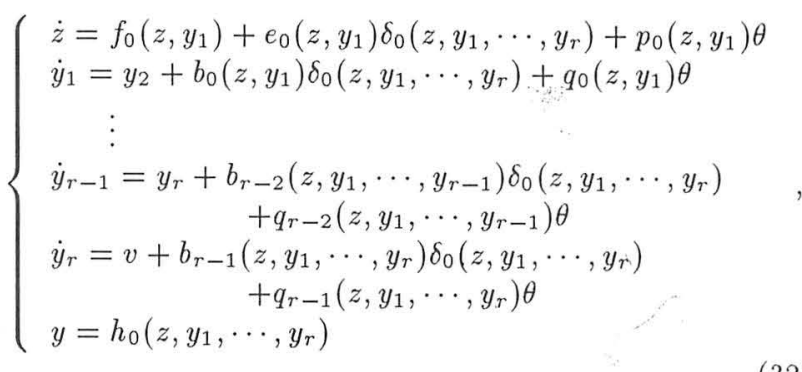

where output $y=y_{1}$.

Let bounding function $n\left(T^{-1}\left(z, y_{1}, \cdots, y_{r}\right)\right)$ for uncertainty $\delta_{0}\left(z, y_{1}, \cdots, y_{r}\right)$ satisfy

$$
\frac{\partial n}{\partial y_{i}}=0, \quad i=2,3, \cdots, r \text {. }
$$

Then, the bounding function is expressed as

$$
\tilde{n}_{0}\left(z, y_{1}\right)=n\left(T^{-1}\left(z, y_{1}, \cdots, y_{r}\right)\right) .
$$

Again for the zero dynamics of (32), assume the following conditions.

A4 Zero dynamics are not influenced by unknown parameter, i.e. $p_{*}(z)=0$.

A5 System (32) has robust minimum phase property, i.e. there exist positive definite functions $V_{0}(z)>$ $0\left(V_{0}(0)=0\right)$ and $Q_{0}(z)>0\left(Q_{0}(0)=0\right)$ such that

$$
L_{f_{*}} V_{0}(z)+L_{e_{*}} V_{0}(z) \delta_{0}\left(z, y_{1}, \cdots, y_{r}\right) \leq-Q_{0}(z)
$$

is satisfied.

For any $1<i<r$ (in order from $i=2$ ), extract $\left(z, y_{1}, \cdots, y_{i}\right)$-subsystem from system (32)

$$
\left\{\begin{array}{c}
\dot{z}=f_{0}\left(z, y_{1}\right)+e_{0}\left(z, y_{1}\right) \delta_{0}\left(z, y_{1}, \cdots, y_{r}\right)+p_{0}\left(z, y_{1}\right) \theta \\
\dot{y}_{1}=y_{2}+b_{0}\left(z, y_{1}\right) \delta_{0}\left(z, y_{1}, \cdots, y_{r}\right)+q_{0}\left(z, y_{1}\right) \theta \\
\dot{y}_{2}=y_{3}+b_{1}\left(z, y_{1}, y_{2}\right) \delta_{0}\left(z, y_{1}, \cdots, y_{r}\right)+q_{1}\left(z, y_{1}, y_{2}\right) \theta \\
\vdots \\
\dot{y}_{i}=y_{i+1}+b_{i-1}\left(z, y_{1}, \cdots, y_{i}\right) \delta_{0}\left(z, y_{1}, \cdots, y_{r}\right) \\
+q_{i-1}\left(z, y_{1}, \cdots, y_{i}\right) \theta
\end{array}\right.
$$

Then, for the subsystem, repeat the control design procedure for systems with relative degree $1 i$ times, and implement change of coordinate

$$
\left\{\begin{array}{rl}
\xi_{i-1} & =\left[\begin{array}{l}
\xi_{i-2} \\
\eta_{i-2}
\end{array}\right] \\
\eta_{i-1} & =y_{i}-\alpha_{i-1}\left(\xi_{i-1}, \hat{\theta}\right)
\end{array},\right.
$$

finally $\left(\xi_{i-1}, \eta_{i-1}\right)$-subsystem is obtained as follows.

$$
\left\{\begin{aligned}
\dot{\xi}_{i-1}=F_{i-1}( & \left.\xi_{i-1}, \hat{\theta}\right)+P_{i-1}\left(\xi_{i-1}, \hat{\theta}\right) \theta \\
& +E_{i-1}\left(\xi_{i-1}, \hat{\theta}\right) \delta_{0}\left(z, y_{1}, \cdots, y_{r}\right) \\
& +G_{i-1}\left(\xi_{i-1}\right) \eta_{i-1}+H_{i-1}\left(\xi_{i-1}, \hat{\theta}\right) \dot{\hat{\theta}} \\
\dot{\eta}_{i-1}=y_{i+1}- & v_{i}\left(\xi_{i-1}, y_{i}, \hat{\theta}\right)+w_{i}\left(\xi_{i-1}, y_{i}, \hat{\theta}\right) \theta \\
& +z_{i}\left(\xi_{i-1}, y_{i}, \hat{\theta}\right) \delta_{0}\left(z, y_{1}, \cdots, y_{r}\right)-\frac{\partial \alpha_{i-1}}{\partial \hat{\theta}} \dot{\hat{\theta}}
\end{aligned}\right.
$$


where

$$
\begin{aligned}
v_{i}\left(\xi_{i-1}, y_{i}, \hat{\theta}\right)= & \frac{\partial \alpha_{i-1}}{\partial z} f_{0}\left(\xi_{1}\right)+\sum_{k=1}^{i-1} \frac{\partial \alpha_{i-1}}{\partial y_{k}} y_{k+1}, \\
w_{i}\left(\xi_{i-1}, y_{i}, \hat{\theta}\right)= & q_{i-1}\left(\xi_{i-1}, y_{i}\right) \\
& -\sum_{k=1}^{i-1} \frac{\partial \alpha_{i-1}}{\partial y_{k}} q_{k-1}\left(\xi_{k}\right)-\frac{\partial \alpha_{i-1}}{\partial z} p_{0}\left(\xi_{1}\right), \\
z_{i}\left(\xi_{i-1}, y_{i}, \hat{\theta}\right)= & b_{i-1}\left(\xi_{i-1}, y_{i}\right) \\
& -\sum_{k=1}^{i-1} \frac{\partial \alpha_{i-1}}{\partial y_{k}} b_{k-1}\left(\xi_{k}\right)-\frac{\partial \alpha_{i-1}}{\partial z} e_{0}\left(\xi_{1}\right) .
\end{aligned}
$$

By taking $y_{i+1}=\alpha_{i}\left(\xi_{i-1}, y_{i}, \hat{\theta}\right)+\eta_{i}$, the closed-loop system of (37) is described as

$$
\begin{aligned}
\dot{\xi}_{i} & =F_{i}\left(\xi_{i}, \hat{\theta}\right)+P_{i}\left(\xi_{i}, \hat{\theta}\right) \theta \\
& +E_{i}\left(\xi_{i}, \hat{\theta}\right) \delta_{0}\left(z, y_{1}, \cdots, y_{r}\right)+G_{i}\left(\xi_{i}\right) \eta_{i}+H_{i}\left(\xi_{i}, \hat{\theta}\right) \dot{\hat{\theta}},(38)
\end{aligned}
$$

where $\xi_{i}=\left[\begin{array}{ll}\xi_{i-1}^{T} & \eta_{i-1}^{T}\end{array}\right]^{T}$,

$$
F_{i}\left(\xi_{i}, \hat{\theta}\right)=\left[\begin{array}{c}
F_{i-1}\left(\xi_{i-1}, \hat{\theta}\right)+G_{i-1}\left(\xi_{i-1}\right) \eta_{i-1} \\
\alpha_{i}\left(\xi_{i}, \hat{\theta}\right)-v_{i}\left(\xi_{i}, \hat{\theta}\right)
\end{array}\right],
$$

$P_{i}\left(\xi_{i}, \hat{\theta}\right)=\left[\begin{array}{c}P_{i-1}\left(\xi_{i-1}, \hat{\theta}\right) \\ w_{i}\left(\xi_{i}, \hat{\theta}\right)\end{array}\right], E_{i}\left(\xi_{i}, \hat{\theta}\right)=\left[\begin{array}{c}E_{i-1}\left(\xi_{i-1}, \hat{\theta}\right) \\ z_{i}\left(\xi_{i}, \hat{\theta}\right)\end{array}\right]$,

$$
G_{i}\left(\xi_{i}\right)=\left[\begin{array}{l}
0 \\
1
\end{array}\right], H_{i}\left(\xi_{i}, \hat{\theta}\right)=\left[\begin{array}{c}
H_{i-1}\left(\xi_{i-1}, \hat{\theta}\right) \\
-\frac{\partial \alpha_{i-1}}{\partial \dot{\theta}}
\end{array}\right] .
$$

Define positive definite functions $V_{i}$ and $Q_{i}$ as

$$
\begin{aligned}
V_{i}\left(\xi_{i-1}, \eta_{i-1}, \tilde{\theta}\right) & =V_{i-1}\left(\xi_{i-1}, \tilde{\theta}\right)+\frac{1}{2} \eta_{i-1}^{T} \eta_{i-1} \\
Q_{i}\left(\xi_{i-1}, \eta_{i-1}\right) & =Q_{i-1}\left(\xi_{i-1}\right)+\frac{1}{2} \eta_{i-1}^{T} \eta_{i-1}
\end{aligned}
$$

Then, the same as derivation of (21), the following inequality is derived.

$$
\begin{aligned}
& \frac{\partial V_{i}}{\partial \xi_{i}}\left\{F_{i}\left(\xi_{i}, \hat{\theta}\right)+P_{i}\left(\xi_{i}, \hat{\theta}\right) \theta\right\}+\frac{\lambda_{i}\left(\xi_{i}\right)}{2}\left\|L_{E_{i}}^{T} V_{i}\left(\xi_{i}\right)\right\|^{2} \\
& \quad+\frac{1}{2 \lambda_{i}\left(\xi_{i}\right)}\left\|\tilde{n}_{0}\left(z, y_{1}\right)\right\|^{2} \\
&=\frac{\partial V_{i-1}}{\partial \xi_{i-1}}\left\{F_{i-1}\left(\xi_{i-1}, \hat{\theta}\right)+P_{i-1}\left(\xi_{i-1}\right) \theta\right\} \\
&+\frac{\lambda_{i}\left(\xi_{i}\right)}{2}\left\|L_{E_{i-1}}^{T} V_{i-1}\left(\xi_{i-1}\right)\right\|^{2}+\frac{1}{2 \lambda_{i}\left(\xi_{i}\right)}\left\|\tilde{n}_{0}\left(z, y_{1}\right)\right\|^{2} \\
&+\eta_{i-1}^{T}\left[\alpha_{i}\left(\xi_{i}, \hat{\theta}\right)+\eta_{i-2}-v_{i}\left(\xi_{i}, \hat{\theta}\right)+w_{i}\left(\xi_{i}, \hat{\theta}\right) \hat{\theta}\right. \\
&+\lambda_{i}\left(\xi_{i}\right)\left\{\frac{1}{2} z_{i}\left(\xi_{i}, \hat{\theta}\right) z_{i}^{T}\left(\xi_{i}, \hat{\theta}\right) \eta_{i-1}\right. \\
&\left.\left.+z_{i}\left(\xi_{i}, \hat{\theta}\right) E_{i-1}^{T}\left(\xi_{i-1}\right) \frac{\partial^{T} V_{i-1}}{\partial \xi_{i-1}}\right\}\right]+\eta_{i-1}^{T} w_{i}\left(\xi_{i}, \hat{\theta}\right) \tilde{\theta} .
\end{aligned}
$$

Choose $\alpha_{i}$ as

$$
\begin{aligned}
\alpha_{i}\left(\xi_{i}, \hat{\theta}\right) & \\
= & -\eta_{i-2}-\frac{1}{2} \eta_{i-1}+v_{i}\left(\xi_{i}, \hat{\theta}\right)-w_{i}\left(\xi_{i}, \hat{\theta}\right) \hat{\theta} \\
& -\lambda_{i}\left(\xi_{i}\right)\left\{\frac{1}{2} z_{i}\left(\xi_{i}, \hat{\theta}\right) z_{i}^{T}\left(\xi_{i}, \hat{\theta}\right) \eta_{i-1}\right. \\
& \left.+z_{i}\left(\xi_{i}, \hat{\theta}\right) E_{i-1}^{T}\left(\xi_{i-1}\right) \frac{\partial^{T} V_{i-1}}{\partial \xi_{i-1}}\right\}+\nu_{i}\left(\xi_{i}, \hat{\theta}\right),
\end{aligned}
$$

and $\lambda_{i}$ as $\lambda_{i}\left(\xi_{i}\right)=\lambda_{i-1}\left(\xi_{i-1}\right)$, then using the result in former step, the following inequality is obtained.

$$
\begin{aligned}
\frac{\partial V_{i}}{\partial \xi_{i}}\left\{F_{i}\left(\xi_{i}, \hat{\theta}\right)+P_{i}\left(\xi_{i}, \hat{\theta}\right) \theta\right\}+\frac{\lambda_{i}\left(\xi_{i}\right)}{2}\left\|L_{E_{i}}^{T} V_{i}\left(\xi_{i}\right)\right\|^{2} \\
+\frac{1}{2 \lambda_{i}\left(\xi_{i}\right)}\left\|\tilde{n}_{0}\left(z, y_{1}\right)\right\|^{2} \\
\leq-Q_{i}\left(\xi_{i-1}, \eta_{i-1}\right)+\tilde{\theta}^{T} \tau_{i}\left(\xi_{i}, \hat{\theta}\right) \\
+\left(\sum_{k=1}^{i-2} \eta_{k}^{T} \frac{\partial \alpha_{k}}{\partial \hat{\theta}}\right) \tau_{i-1}\left(\xi_{i-1}, \hat{\theta}\right)+\eta_{i-1}^{T} \nu_{i}\left(\xi_{i}, \hat{\theta}\right)
\end{aligned}
$$

where

$$
\tau_{i}\left(\xi_{i}, \hat{\theta}\right)=\tau_{i-1}\left(\xi_{i-1}\right)+w_{i}^{T}\left(\xi_{i}, \hat{\theta}\right) \eta_{i-1} .
$$

Thus, (41) can be rewritten as

$$
\begin{aligned}
& \frac{\partial V_{i}}{\partial \xi_{i}}\left\{F_{i}\left(\xi_{i}, \hat{\theta}\right)+P_{i}\left(\xi_{i}, \hat{\theta}\right) \theta\right\}+\frac{\lambda_{i}\left(\xi_{i}\right)}{2}\left\|L_{E_{i}}^{T} V_{i}\left(\xi_{i}\right)\right\|^{2} \\
& \quad+\frac{1}{2 \lambda_{i}\left(\xi_{i}\right)}\left\|\tilde{n}_{0}\left(z, y_{1}\right)\right\|^{2} \\
& \leq-Q_{i}\left(\xi_{i-1}, \eta_{i-1}\right)+\tilde{\theta}^{T} \tau_{i}\left(\xi_{i}, \hat{\theta}\right)+\left(\sum_{k=1}^{i-2} \eta_{k}^{T} \frac{\partial \alpha_{k}}{\partial \hat{\theta}}\right) \tau_{i}\left(\xi_{i}, \hat{\theta}\right) \\
& \quad-\left(\sum_{k=1}^{i-2} \eta_{k}^{T} \frac{\partial \alpha_{k}}{\partial \hat{\theta}}\right) w_{i}^{T}\left(\xi_{i}, \hat{\theta}\right) \eta_{i-1}+\eta_{i-1}^{T} \nu_{i}\left(\xi_{i}, \hat{\theta}\right) .
\end{aligned}
$$

Consider the time derivative of $V_{i}\left(\xi_{i-1}, \eta_{i-1}, \tilde{\theta}\right)$ along the trajectory of (38), and substituting (43), the following inequality is obtained.

$$
\begin{aligned}
\dot{V}_{i}= & \frac{\partial V_{i}}{\partial \xi_{i}}\left\{F_{i}\left(\xi_{i}, \hat{\theta}\right)+P_{i}\left(\xi_{i}, \hat{\theta}\right) \theta\right\} \\
& +\frac{\partial V_{i}}{\partial \xi_{i}}\left\{E_{i}\left(\xi_{i}, \hat{\theta}\right) \delta_{0}\left(z, y_{1}, \cdots, y_{r}\right)\right. \\
& \left.+G_{i}\left(\xi_{i}\right) \eta_{i}+H_{i}\left(\xi_{i}, \hat{\theta}\right) \dot{\hat{\theta}}\right\}+\tilde{\theta}^{T} \dot{\tilde{\theta}} \\
\leq & -Q_{i}\left(\xi_{i-1}, \eta_{i-1}\right) \\
& -\frac{\lambda_{i}\left(\xi_{i}\right)}{2}\left\|L_{E_{i}}^{T} V_{i}\left(\xi_{i}\right)-\frac{1}{\lambda_{i}\left(\xi_{i}\right)} \delta_{0}\left(z, y_{1}, \cdots, y_{r}\right)\right\|^{2} \\
& -\frac{1}{2 \lambda_{i}\left(\xi_{i}\right)}\left\{\left\|\tilde{n}_{0}\left(z, y_{1}\right)\right\|^{2}-\left\|\delta_{0}\left(z, y_{1}, \cdots, y_{r}\right)\right\|^{2}\right\}+\eta_{i-1}^{T} \eta_{i} \\
& +\tilde{\theta}^{T}\left[\tau_{i}\left(\xi_{i}, \hat{\theta}\right)-\dot{\hat{\theta}}\right]+\left(\sum_{k=1}^{i-2} \eta_{k}^{T} \frac{\partial \alpha_{k}}{\partial \hat{\theta}}\right) \tau_{i}\left(\xi_{i}, \hat{\theta}\right) \\
& -\left(\sum_{k=1}^{i-2} \eta_{k}^{T} \frac{\partial \alpha_{k}}{\partial \hat{\theta}}\right) w_{i}^{T}\left(\xi_{i}, \hat{\theta}\right) \eta_{i-1}+\eta_{i-1}^{T} \nu_{i}\left(\xi_{i}, \hat{\theta}\right) \\
& -\left(\sum_{k=1}^{i-1} \eta_{k}^{T} \frac{\partial \alpha_{k}}{\partial \hat{\theta}}\right) \dot{\hat{\theta}} .
\end{aligned}
$$

By choosing $\nu_{i}$ as

$\nu_{i}\left(\xi_{i}, \hat{\theta}\right)=w_{i}\left(\xi_{i}, \hat{\theta}\right)\left(\sum_{k=1}^{i-2} \frac{\partial^{T} \alpha_{k}}{\partial \hat{\theta}} \eta_{k}\right)+\frac{\partial \alpha_{i-1}}{\partial \hat{\theta}} \tau_{i}\left(\xi_{i}, \hat{\theta}\right)$,

finally the following inequality holds.

$$
\begin{aligned}
\dot{V}_{i} \leq & -Q_{i}\left(\xi_{i-1}, \eta_{i-1}\right)+\tilde{\theta}^{T}\left[\tau_{i}\left(\xi_{i}, \hat{\theta}\right)-\dot{\hat{\theta}}\right] \\
& +\left(\sum_{k=1}^{i-1} \eta_{k}^{T} \frac{\partial \alpha_{k}}{\partial \hat{\theta}}\right)\left[\tau_{i}\left(\xi_{i}, \hat{\theta}\right)-\dot{\hat{\theta}}\right]+\eta_{i-1}^{T} \eta_{i} .
\end{aligned}
$$


Therefore, if parameter update law is chosen as $\hat{\theta}=$ $\tau_{i}\left(\xi_{i}, \hat{\theta}\right)$, the closed-loop system from input $\eta_{i}$ to output $\eta_{i-1}$ becomes strictly robust passive.

In this way, in $i+1$-th step, repeat the same procedure for $\left(\xi_{i}, \eta_{i}\right)$-subsystem, and construct a Lyapunov function $V_{i+1}$. Finally, by repeating this procedure $r$ times, robust adaptive stabilizing controller for whole system can be obtained. In fact, in the $r$-th repetition, take $\eta_{r}=0$ and choosing parameter update law as

$$
\dot{\hat{\theta}}=\tau_{r}\left(\xi_{r}, \hat{\theta}\right),
$$

then, for the $r$-th Lyapunov function, the following inequality holds.

$$
\dot{V}_{r}<0, \quad \forall \xi_{r} \neq 0
$$

\section{Conclusions}

In this paper, we extended a design method of the robust adaptive stabilizing controller for uncertain nonlinear systems with relative degree 1 proposed in [5] to the uncertain nonlinear systems with arbitrary relative degree. We provided some conditions which nonlinear systems should satisfy for the sake of the problem to be solvable, and showed that under the conditions, the controller can be obtained using control scheme in [5] recursively. By adding some suitable conditions, it is possible to extend our results to MIMO systems.

\section{Appendix}

Definition 1: (Relative Degree) The nonlinear system (1) is said to have relative degree $r$ if (i) $L_{g} L_{f}^{k} h(x)=0,{ }^{\forall} x$ for all $k<r-1$ and (ii) $L_{g} L_{f}^{r-1} h(x) \neq 0,{ }^{\forall} x$.

Definition 2: (Strict Robust Passivity) The nonlinear system (1) is said to be strictly robust passive if there exist positive semi-definite function $V(x) \geq 0$ and positive definite function $Q(x)>0$ such that $\dot{V} \leq u^{T} y-Q(x), \forall u$ is satisfied for all $\delta \in \Omega$.

Lemma 1: ${ }^{[5]} \quad$ Consider functions $\beta: R^{n} \rightarrow R^{q_{1}}$ and $n: R^{n} \rightarrow R^{q_{2}}$, negative definite function $\alpha: R^{n} \rightarrow R$ and positive definite function $Q: R^{n} \rightarrow R$. For all $\delta \in \Omega$, inequality

$$
\alpha(x)+\beta^{T}(x) \delta(x) \leq-Q(x), \quad \forall x
$$

holds if and only if there exists positive definite function $\lambda(x)$ such that

$$
\alpha(x)+\frac{\lambda(x)}{2}\left\|\beta^{T}(x)\right\|^{2}+\frac{1}{2 \lambda(x)}\|n(x)\|^{2} \leq-Q(x), \quad \forall x
$$

holds.

Lemma 2: ${ }^{[5]}$ Consider SISO nonlinear system given by

$$
\left\{\begin{array}{l}
\dot{z}=f_{0}(z, y)+e_{0}(z, y) \delta_{0}(z, y)+p_{0}(z, y) \theta \\
\dot{y}=v+b_{0}(z, y) \delta_{0}(z, y)+q_{0}(z, y) \theta \\
y=h_{0}(z, y)
\end{array},\right.
$$

where $\delta_{0}$ satisfies $\left\|\delta_{0}(z, y)\right\|^{2} \leq\left\|n_{0}(z, y)\right\|^{2}$.

Assume that $p_{0}(z, 0)=0$ and system (51) has robust minimum phase property, i.e. there exist positive definite functions $V(z)>0(V(0)=0)$ and $Q(z)>0$ $(Q(0)=0)$ such that

$$
L_{f_{*}} V(z)+L_{e_{*}} V(z) \delta_{*}(z) \leq-Q(z)
$$

is satisfied for all $\delta_{0}$. Smooth functions $f_{0}, \frac{\partial V}{\partial z} e_{0}$, $\frac{\partial V}{\partial z} p_{0}$ and $n_{0}$ are decomposed ${ }^{[5]}$ as

$$
\begin{aligned}
f_{0}(z, y) & =f_{*}(z)+f_{1}(z, y) y, \\
\frac{\partial V}{\partial z} e_{0}(z, y) & =\frac{\partial V}{\partial z} e_{*}(z)+y^{T} e_{1}(z, y), \\
\frac{\partial V}{\partial z} p_{0}(z, y) & =\frac{\partial V}{\partial z} p_{*}(z)+y^{T} p_{1}(z, y), \\
n_{0}(z, y) & =n_{*}(z)+n_{1}(z, y) y .
\end{aligned}
$$

Then, robust regulation problem such taht the closedloop system achieves $\lim _{t \rightarrow \infty} y(t)=0$ for all $\delta_{0}$ is solvable, and robust adaptive controller is given by

$$
\left\{\begin{array}{l}
v=-c_{0}(z, y, \hat{\theta})-\frac{1}{2} y \\
\dot{\hat{\theta}}=\left\{p_{1}^{T}(z, y)+q_{0}^{T}(z, y)\right\} y
\end{array},\right.
$$

where

$$
\begin{gathered}
c_{0}(z, y, \hat{\theta})=c_{1}(z, y) \frac{\partial^{T} V}{\partial z}+c_{2}(z, y) \hat{\theta}+c_{3}(z, y), \\
c_{1}(z, y)=f_{1}^{T}(z, y)+\lambda(z)\left\{e_{1}(z, y)+b_{0}(z, y)\right\} e_{*}^{T}(z), \\
c_{2}(z, y)=p_{1}(z, y)+q_{0}(z, y), \\
c_{3}(z, y)=\frac{1}{\lambda(z)} n_{1}^{T}(z, y)\left\{n_{*}(z)+\frac{1}{2} n_{1}(z, y) y\right\} \\
+\frac{\lambda(z)}{2}\left\{e_{1}(z, y)+b_{0}(z, y)\right\}\left\{e_{1}^{T}(z, y)+b_{0}^{T}(z, y)\right\} y \cdot \text {. }
\end{gathered}
$$

\section{References}

[1] M. Kristić and H. Deng: Stabilization of Nonlinear Systems with uncertainties, Preprints of the First Nonlinear Control Network, Pedagogical School, pp.203-243, 1999.

[2] D. J. Hill and P. J. Moylan: The Nonlinear Dissipative Systems, IEEE Trans. Automa. Contr., Vol.21, pp.708-711, 1976.

[3] C. I. Byrnes, A. Isidori and J. C. Willems: Passivity, feedback equivalence, and the global stabilization of minimum phase nonlinear systems, IEEE Trans. Automa. Contr., Vol.36, pp.1228-1240, 1991.

[4] M. Kristić, I. Kanellakopoulos and P. Kokotović: Nonlinear and Adaptive Control Design, WILLEYINTERSCIENCE, 1995.

[5] T. Shen, Y. Mutoh and K. Tamura: A Design Method of Adaptive Robust Controller for Nonlinear Systems -with Modelling Errors, Trans. SICE, Vol.34, No.10, pp.1388-1394, 1998. (in Japanese)

[6] C. Ishii and T. Shen: Robust Adaptive Control with Disturbance Attenuation for Nonlinear Systems with Uncertainty, Proc. of the ACC, 2002. (submitted) 